\title{
Effect of Ruboxistaurin on the Visual Acuity Decline Associated with Long-standing Diabetic Macular Edema
}

\section{Citation}

Davis, Matthew D., Matthew J. Sheetz, Lloyd P. Aiello, Roy C. Milton, Ronald P. Danis, Xin Zhi, Aniz Girach, Maria C. Jimenez, and Louis Vignati. 2009. Effect of Ruboxistaurin on the Visual Acuity Decline Associated with Long-Standing Diabetic Macular Edema. Invest. Ophthalmol. Vis. Sci. 50, no. 1: 1. doi:10.1167/iovs.08-2473.

\section{Published Version}

doi:http:10.1167/iovs.08-2473

\section{Permanent link}

http://nrs.harvard.edu/urn-3:HUL.InstRepos:33776277

\section{Terms of Use}

This article was downloaded from Harvard University's DASH repository, and is made available under the terms and conditions applicable to Other Posted Material, as set forth at http:// nrs.harvard.edu/urn-3:HUL.InstRepos:dash.current.terms-of-use\#LAA

\section{Share Your Story}

The Harvard community has made this article openly available.

Please share how this access benefits you. Submit a story.

Accessibility 


\title{
Effect of Ruboxistaurin on the Visual Acuity Decline Associated with Long-standing Diabetic Macular Edema
}

\author{
Matthew D. Davis, ${ }^{1}$ Mattbew J. Sheetz, ${ }^{2}$ Lloyd P. Aiello, ${ }^{3}$ Roy C. Milton, ${ }^{4}$ Ronald P. Danis, ${ }^{1}$ \\ Xin Zhi, ${ }^{2}$ Aniz Girach, ${ }^{5}$ Maria C. Jimenez, ${ }^{2}$ and Louis Vignati, ${ }^{2}$ for the \\ PKC-DRS2 Study Group
}

Purpose. To compare relationships between severity and duration of diabetic macular edema (DME) and visual acuity (VA) observed in the PKC-DRS2 with those from the Early Treatment Diabetic Retinopathy Study (ETDRS) and to assess the effect of the orally administered PKC $\beta$ inhibitor ruboxistaurin (RBX) on these parameters.

Methods. In the PKC-DRS2, patients with moderately severe to very severe nonproliferative diabetic retinopathy $(n=685)$ were randomly assigned to $32 \mathrm{mg} / \mathrm{d}$ RBX or placebo and followed up for 36 months with ETDRS VA measurements and fundus photographs (FP) every 3 to 6 months. Mean VA was calculated across all FP visits for eyes in each level of the ETDRS DME severity scale at those visits. For eyes with baseline VA $\geq 20 / 40$, relationships between change in VA from baseline to last visit and duration of severe DME were analyzed with linear regression.

Results. Mean VA decreased by approximately 22 letters between the mildest and most severe levels of the DME scale in the PKC-DRS2, compared with 27 letters in the ETDRS. In the placebo group, the rate of decrease in VA over time associated with duration of severe DME was 0.67 letters per month (24 letters over 36 months, compared with 20 letters over 28-36 months in the ETDRS). This rate was 30\% less in the RBX group (0.47 letter per month, $P=0.022$ ).

Conclusions. The VA decrease in the PKC-DRS2 associated with long-standing DME agrees well with estimates from the ETDRS. RBX appears to ameliorate this decrease, an effect that could be important clinically. (ClinicalTrials.gov number, NCT00604383.) (Invest Ophthalmol Vis Sci. 2009;50:1-4) DOI:10.1167/iovs.082473

From the ${ }^{1}$ Department of Ophthalmology and Visual Sciences, University of Wisconsin, Madison, Wisconsin; ${ }^{2}$ Lilly Research Laboratories, Indianapolis, Indiana; the ${ }^{3}$ Beetham Eye Institute, Joslin Diabetes Center and Department of Ophthalmology, Harvard University Medical School, Boston, Massachusetts; ${ }^{4}$ EMMES Corporation, Rockville, Maryland; and ${ }^{5}$ Lilly Research Laboratories, Windlesham, Surrey, United Kingdom.

Supported by Eli Lilly \& Company.

Submitted for publication June 23, 2008; revised August 1, 2008; accepted October 21, 2008.

Disclosure: M.D. Davis, Eli Lilly \& Company (F); M.J. Sheetz, Eli Lilly \& Company (E); L.P. Aiello, Eli Lilly \& Company (F); R.C. Milton, Eli Lilly \& Company (F); R.P. Danis, None; X. Zhi, Eli Lilly \& Company (E); A. Girach, Eli Lilly \& Company (E); M.C. Jimenez, Eli Lilly \& Company (E); L. Vignati, Eli Lilly \& Company (E)

The publication costs of this article were defrayed in part by page charge payment. This article must therefore be marked "advertisement" in accordance with 18 U.S.C. $\$ 1734$ solely to indicate this fact.

Corresponding author: Matthew J. Sheetz, Lilly Research Laboratories, Eli Lilly Corporate Center, Indianapolis, IN 46285; msheetz@lilly.com.
$\mathrm{D}$ iabetic retinopathy (DR) and diabetic macular edema (DME) are common complications of diabetes mellitus and frequent causes of visual impairment. ${ }^{1}$ DME features excessive permeability of retinal capillaries and inadequate fluid reabsorption leading to accumulation of fluid in the macula, sometimes resulting in retinal thickening at the macular center. ${ }^{2}$ Many of the microvascular alterations in the eyes of patients with diabetes are thought to arise from hyperglycemiainduced activation of protein kinase C (PKC). Preclinical models of diabetes have demonstrated that activation of the PKC $\beta$ isoform results in increased neovascularization and retinal vascular permeability ${ }^{3-5}$ which can be reduced with oral administration of ruboxistaurin (RBX), an isoform-specific inhibitor of PKC $\beta .^{6-8}$ RBX has also been shown to normalize retinal circulation in patients with diabetes.

In the Protein Kinase $\mathrm{C} \beta$ Inhibitor-Diabetic Retinopathy Study 2 (PKC-DRS2), oral administration of RBX $(32 \mathrm{mg} / \mathrm{d}$ ) reduced sustained moderate visual loss (SMVL; $\geq 1$ 15-letter loss on the Early Treatment Diabetic Retinopathy Study (ETDRS) chart sustained from months 30 through 36 or for the last 6 months of study participation in those who discontinued early) in patients with moderately severe to very severe nonproliferative diabetic retinopathy (NPDR) from $9.1 \%$ in placebo-treated patients to $5.5 \%$ in RBX-treated patients. ${ }^{10}$ Treatment with RBX was also associated with less progression of DME to within 100 $\mu \mathrm{m}$ of the macular center in eyes of patients who had clinically significant macular edema (CSME) at baseline and with less frequent initial application of photocoagulation for DME in study eyes without prior history of such treatment at baseline. ${ }^{10}$ In the PKC-Diabetic Macular Edema Study, which was designed to test the effect of RBX on non-center-involved DME, RBX treatment also showed a beneficial effect on DME progression relative to placebo. ${ }^{11}$

In previous reports regarding $\mathrm{RBX}$ treatment, morphologic changes in DME severity and changes in VA have been analyzed separately. In this study, our objective was to use the recently developed ETDRS DME severity scale ${ }^{12}$ to assess relationships between severity and duration of DME and VA and to examine the effect of RBX treatment on them. We chose the PKC-DRS2 for these analyses because it included eyes with a broad range of DME severity. Even so, the number of eyes in which severe DME was observed for long periods was small. The outcomes reported herein are not the primary endpoints of the trial.

\section{MethodS}

\section{Study Design}

The study design and methods have been published previously. ${ }^{10}$ Briefly, the PKC-DRS2 was a multicenter, parallel, placebo-controlled, double-masked clinical trial. Patients in the PKC-DRS2 were randomly assigned to receive $\mathrm{RBX} 32 \mathrm{mg} / \mathrm{d}(n=345)$ or placebo $(n=340)$ orally 
and were observed for 36 to 42 months. The study was conducted at 70 investigative sites in the United States.

The study was conducted in accordance with the ethical principles stated in the Declaration of Helsinki and guidelines for good clinical practices. Approval of the study protocol by the ethics review board of each study site, and signed informed consent from each patient enrolled was obtained before the studies began.

\section{Patients}

The main inclusion criteria were (1) diagnosis of type 1 or 2 diabetes, (2) age $\geq 18$ years, (3) hemoglobin $A_{1 C} \leq 13 \%$, (4) no previous history of panretinal photocoagulation, (5) best corrected VA score of 45 or more letters on the ETDRS chart $(\sim 20 / 120$ or better $),{ }^{13}$ and (5) moderately severe to very severe NPDR (ETDRS retinopathy levels $\geq 47 \mathrm{~A}$ and $\leq 53 \mathrm{E}) .{ }^{14}$ Any level of DME was allowed, including none. All patients had to have at least one eye that met the entry criteria. If the fellow eye had retinopathy severity less than proliferative DR without prior panretinal photocoagulation, both eyes were considered to be study eyes. Otherwise, only the eligible eye was considered to be a study eye. Only data from study eyes were analyzed.

\section{Ophthalmic Evaluation}

Best corrected VA was measured with the ETDRS protocol ${ }^{13}$ at screening and at each visit. Ophthalmic examination performed at each visit included ophthalmoscopy, slit lamp biomicroscopy, Age-Related Eye Disease Study (AREDS) clinical lens grading, and measurement of intraocular pressure. Stereoscopic fundus photographs were taken at baseline, 3 and 6 months, and every 6 months thereafter.

\section{ETDRS DME Severity Scale}

The scale was constructed by cross-classifying the area of retinal thickening within one optic disc diameter (DD) of the macular center by degree of thickening at the center estimated from stereoscopic fundus photographs of each eye of 3711 patients enrolled in the ETDRS. ${ }^{12}$ Mean baseline VA was calculated for the eyes in each of the 24 cells in the table, and cells with similar VA were combined by using cluster analysis to create a scale with five major steps, some of which have subdivisions. Levels of particular interest in our analyses are levels $1 \mathrm{~A}$ and $1 \mathrm{~B}$ combined (no retinal thickening within $1 \mathrm{DD}$ of the macular center) and levels 4 or greater combined (mild to severe retinal thickening at the macular center and 1 to 4 disc areas of retinal thickening within $1 \mathrm{DD}$ of the center). The intermediate levels (1C, 2, 3A, and 3B) had lesser areas of retinal thickening and/or less or no thickening at the macular center.

\section{Statistical Analyses}

Data were analyzed by using a modified intent-to-treat population (all enrolled patients with a baseline and at least one postbaseline measurement of that particular endpoint). Linear regression was used to examine the relationship between duration of DME in ETDRS DME severity level 4 or 5 (designated severe) and baseline-to-endpoint change in VA among eyes with baseline VA score $\geq 70$ letters $(\sim 20 / 40$ or better). The analysis was limited to eyes with baseline VA $\geq 70$ letters because we considered it likely that many eyes with baseline VA $<70$ letters had already had long periods of severe DME before baseline, making our estimates of duration less reliable. At baseline, $45 \%$ of eyes with VA $<70$ letters had severe DME compared with $11 \%$ of eyes with VA $\geq 70$ letters. To define the duration of DME, at each follow-up visit with DME level $\geq 4$, severe DME was assumed to have been present since the most recent follow-up visit with gradable photographs (regardless of DME level at that visit) and all such intervals were summed. The probabilities were not adjusted for multiple comparisons.

\section{RESUlts}

\section{Relationship between DME Severity and VA}

The relationship between DME severity on the ETDRS DME scale and VA is shown in Figure 1. DME severity and VA were assessed cross-sectionally at each visit, without regard to previous values. Mean VA was calculated for all visits at the specified DME level, without regard to duration of DME at that level. Mean VA decreased from approximately 80 letters in level $1 \mathrm{AB}$ to $<60$ letters in level $5 \mathrm{~B}$, and there was little difference between the placebo and RBX-treated groups.

\section{Relationship between Duration of Severe DME and VA}

The relationship between duration of severe DME (ETDRS DME level $\geq 4$ ) and VA was assessed by analyzing the distribution of baseline-to-endpoint change in VA by duration of severe DME during the study among eyes with baseline VA $\geq$ 70 letters. The baseline distributions of ocular factors that might influence this relationship were similar in the placebo and RBX groups (Table 1). In placebo-treated patients (Fig. 2A), there was progressively greater decline in VA with increasing duration of severe DME. There was no decrease in median VA in the 304 eyes that never exhibited severe DME at any visit during follow-up in the trial (8 of these eyes had severe DME at baseline but not at any follow-up visit, as did 14 of the corre-

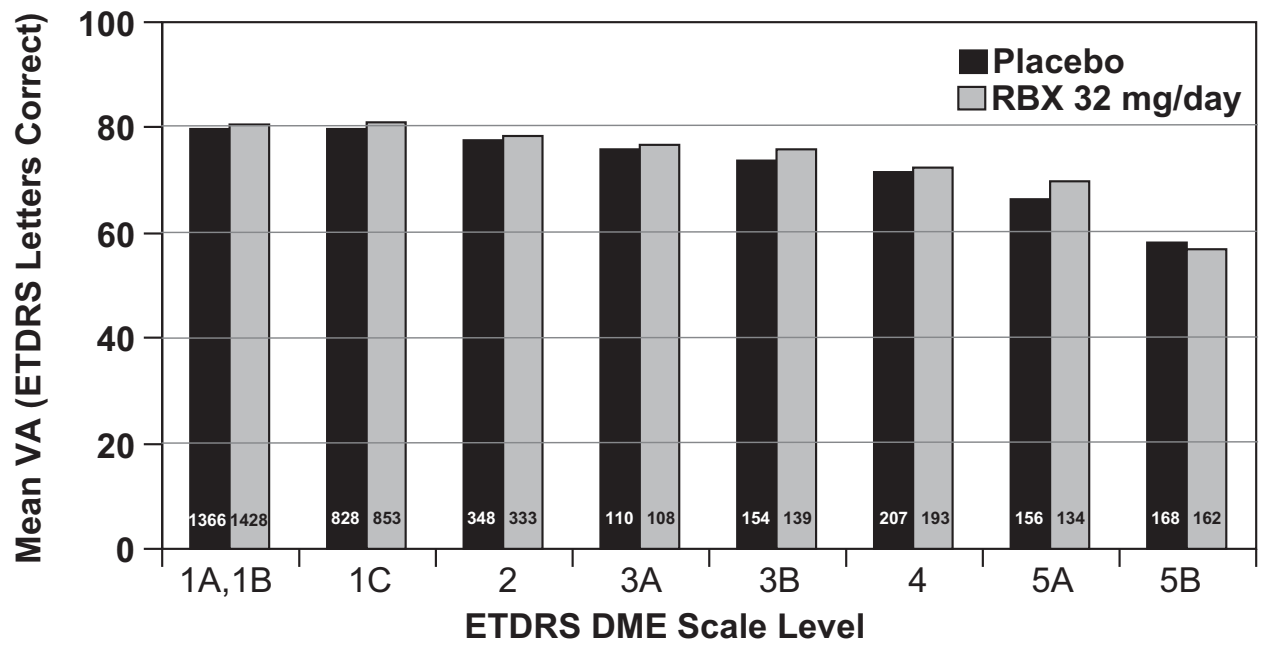

Figure 1. Mean VA in the eyes of placebo- or RBX-treated patients at all follow-up visits by DME severity categorized using the ETDRS DME severity scale. The number of eye-visits at each DME severity level is presented on each bar, and each visit was treated as a different observation. Mean VA (ETDRS letters correct) is reported. 
sponding eyes in the RBX group). In the 161 eyes in the placebo group that had severe DME at one or more follow-up visits, the decline in VA grew progressively greater with longer duration of DME of this severity ( -0.63 letters per month, $P$ for trend $=0.0002)$. Among RBX-treated eyes (Fig. 2B) there were 124 that had severe DME at one or more follow-up visits and in them there appears to have been less decline in VA for a given duration of severe DME $(-0.30$ letters per month, $P$ for trend $=0.0332 ; P=0.010$ for difference in slopes between Figs. 2A, 2B). In similar analyses in eyes with baseline VA $<70$ letters, no relationship between duration of severe DME and VA loss was apparent in either treatment group (data not shown).

The results shown in Figure 2 and just described were not adjusted for baseline factors that might be predictive of baseline-to-endpoint change in VA. In a regression analysis of this outcome that included such factors, as well as duration of severe DME and treatment, there was a strong interaction $(P=$ $0.0019)$ between treatment and duration of severe DME. Accordingly, similar analyses, which included baseline levels for ETDRS DR severity ( $\geq 47 \mathrm{~B}$ vs. $<47 \mathrm{~B}$ ), ETDRS DME severity ( $\geq 4$ vs. $<4$ ), VA (letters), and history of prior photocoagulation for DME, in addition to duration of severe DME, were performed separately in each treatment group. In the placebo group, the estimate for the effect of duration of severe DME was 0.67 letters lost per month of severe DME $(P<0.0001)$, corresponding to 24 letters over the 36-month duration of the trial (74\% of patients completed at least 36 months of follow-up). From baseline to endpoint, on average, eyes with prior photocoagulation for DME lost 2.1 fewer letters than those without $(P=$ 0.073); eyes in ETDRS DR severity level $\geq 47 \mathrm{~B}$ lost 1.7 more letters than those in level $\leq 47 \mathrm{~A}(P=0.13)$, and for every 5-letter decrease in baseline VA, eyes lost 0.8 fewer letters $(P=$ 0.093).

In the RBX group, the estimate for the effect of duration of severe DME was 0.47 letters lost per month of severe DME $(P<0.0001)$, corresponding to 17 letters over the 36-month duration of the trial. $P$ for difference between the placebo and RBX groups in the effect of duration was 0.022 . In this analysis, prior photocoagulation was not prognostic, whereas results for DR severity and baseline VA were similar to those in the placebo group analysis: eyes in level $\geq 47 \mathrm{~B}$ lost 2.0 more letters $(P=0.024)$ and for every 5 -letter decrease in baseline VA, eyes lost 1.1 fewer letters $(P=0.0039)$. In the RBX analysis, unlike the analysis in the placebo group, baseline DME severity level appeared to be a risk factor; eyes in DME level $\geq$

TABLE 1. Baseline Characteristics of Eyes with Baseline $\mathrm{VA} \geq 70$ Letters

\begin{tabular}{|c|c|c|c|}
\hline & Placebo $(n=465)$ & $\operatorname{RBX}(n=461)$ & $P\left(\chi^{2}\right)$ \\
\hline \multicolumn{4}{|l|}{ VA } \\
\hline $95-100$ & $7(1.51)$ & $9(1.95)$ & 0.4023 \\
\hline $90-94$ & $39(8.39)$ & $48(10.41)$ & \\
\hline $85-89$ & $119(25.59)$ & $105(22.78)$ & \\
\hline $80-84$ & $125(26.88)$ & $139(30.15)$ & \\
\hline $75-79$ & $104(22.37)$ & $106(22.99)$ & \\
\hline $70-74$ & $71(15.27)$ & $54(11.71)$ & \\
\hline \multicolumn{4}{|c|}{ ETDRS DME level } \\
\hline 1 & $327(70.47)$ & $316(68.70)$ & 0.856 \\
\hline $2-3$ & $85(18.32)$ & $90(19.57)$ & \\
\hline 4 & $18(3.88)$ & $22(4.78)$ & \\
\hline 5 & $34(7.33)$ & $32(6.96)$ & \\
\hline \multicolumn{4}{|c|}{ DR severity } \\
\hline$\leq 47 \mathrm{~A}$ & $235(50.54)$ & $240(52.06)$ & 0.643 \\
\hline$>47 \mathrm{~A}$ & $230(49.46)$ & $221(47.94)$ & \\
\hline Prior PC & $214(46.02)$ & $192(41.65)$ & 0.180 \\
\hline
\end{tabular}

Data are the number of eyes (\% of total).
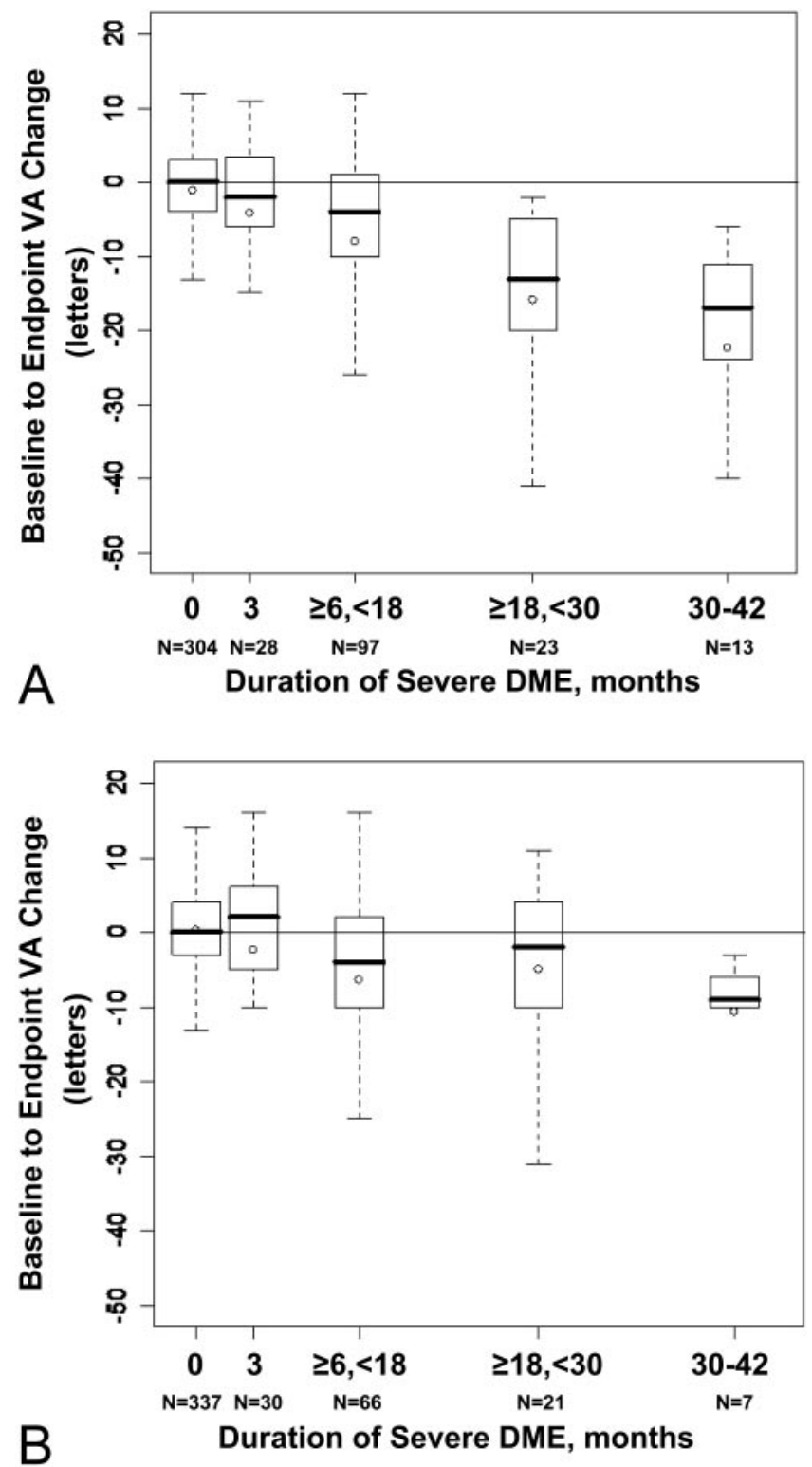

FiguRE 2. Among eyes with baseline visual acuity (VA) $\geq 70$ letters, baseline (BL)-to-endpoint (EP) change in VA by duration of severe DME (ETDRS DME level $\geq 4$ ) in (A) placebo-treated patients and (B) RBXtreated patients. Linear trend for placebo: The slope of -0.627 indicates 0.63 letters lost per month of duration of severe DME, $P=$ 0.0002 . Linear trend for RBX $32 \mathrm{mg} / \mathrm{d}$ : the slope of -0.30 indicates 0.3 letters lost per month of duration of severe DME, $P=0.0332$. Rate of VA decrease in RBX reduced by $52 \%$ compared with placebo, $P=$ 0.010 .

4 at baseline lost 5.7 fewer letters than those in level $<4(P=$ $0.0002)$.

\section{Discussion}

The 20-letter decrease in concurrent VA between levels $1 \mathrm{AB}$ and $5 \mathrm{~B}$ of the ETDRS DME severity scale, in an analysis that did not take duration of DME into account (Fig. 1), was similar to that reported for the baseline data used in developing the scale. ${ }^{12}$ ETDRS analyses using the scale and taking duration of DME into account are, to our knowledge, the first to document the association of decreasing VA with increasing duration of DME, although this association has long been recognized on 
the basis of clinical experience. Our observation of a VA loss of 0.63 letters per month of severe DME in the eyes of placebotreated patients, corresponding to 23 letters over a 36-month period, was in good agreement with the median 4-line $(\sim 20$ letter) loss over a 28- to 36-month period reported in ETDRS eyes. ${ }^{12}$ This agreement, in a data set independent from that used in developing the ETDRS scale, supports the generalizability of the ETDRS findings. In the eyes of RBX-treated patients, the (unadjusted) rate of loss was about one half as great, 0.30 letters per month $(P=0.010$ for the difference between slopes). This difference suggests that RBX may ameliorate the vision-damaging effect of long periods of severe DME.

In placebo-treated patients adjustment for baseline factors (ETDRS DR severity level, VA, ETDRS DME severity level, prior photocoagulation for DME) had little impact on the rate of visual loss with increasing duration of severe DME (0.63 and 0.67 letters lost per month, respectively, in unadjusted and adjusted analyses). In RBX-treated patients, in an analysis adjusted for these same factors, the rate of visual loss changed from 0.30 to 0.47 letters lost per month of severe DME, apparently as a result of an independent effect on eyes in ETDRS DME severity level $\geq 4$ at baseline, which lost 5.7 fewer letters between baseline and endpoint than eyes in less severe levels. In these adjusted analyses, the RBX-treated group had a $30 \%$ decrease in the rate of visual loss per month of duration of severe DME $(P=0.022)$.

If $\mathrm{RBX}$ does provide some degree of protection against the vision-damaging effect of retinal edema, in addition to its effect in reducing retinal thickening, it could be important clinically for eyes in which retinal thickening persists despite treatments intended to reduce it. This protective effect may also be of theoretical interest as a possible manifestation of neuroprotection. ${ }^{15,16}$

The outcomes presented in this report were not the primary endpoints of the trial and were not prospectively defined. There are several additional limitations. Although the number of eyes analyzed in each treatment group was almost 500, approximately 300 in each group never exhibited severe DME during follow-up, and the number with severe DME for 18 months or longer were small (28 and 36, respectively, in the placebo and RBX groups). Patients were randomly assigned to placebo or RBX, but approximately $20 \%$ of study eyes were excluded (those with baseline VA $<70$ letters). However, the groups were well balanced on baseline factors, and bias is unlikely because VA examiners and photograph graders were masked to treatment group. Moreover, our estimate of the rate of VA loss with duration of severe DME in the placebo group is similar to that reported for the sevenfold larger number of eyes in the ETDRS and consistent with the clinical impression that eyes in which severe DME persists for several years are likely to lose several lines of VA.

In summary, concurrent analyses of DME severity and VA in the PKC-DRS2 provide confirmation of ETDRS analyses documenting the increasing VA loss associated with increasing duration of severe DME and suggest that RBX treatment may ameliorate this edema-associated visual decline.

\section{References}

1. Eye Diseases Prevalence Research Group. The prevalence of diabetic retinopathy among adults in the United States. Arch Ophthalmol. 2004;122:552-563.

2. Ferris FL III, Patz A. Macular edema: a complication of diabetic retinopathy. Surv of Opbthalmol. 1984;28:452- 461.

3. Suzuma K, Takahara N, Suzuma I, et al. Characterization of protein kinase $\mathrm{C} \beta$ isoform's action on retinoblastoma protein phosphorylation, vascular endothelial growth factor-induced endothelial cell proliferation, and retinal neovascularization. Proc Natl Acad Sci US A. 2002;99:721-726.

4. Ishii H, Koya D, King GL. Protein kinase C activation and its role in the development of vascular complications in diabetes mellitus. $J$ Mol Med. 1997;76:21-31.

5. Xia P, Aiello LP, Ishii H, et al. Characterization of vascular endothelial growth factor's effect on the activation of protein kinase C, its isoforms, and endothelial cell growth. J Clin Invest. 1996;98: 2018-2026.

6. Aiello LP, Bursell SE, Clermont A, et al. Vascular endothelial growth factor-induced retinal permeability is mediated by protein kinase $\mathrm{C}$ in vivo and suppressed by an orally effective $\beta$-isoformselective inhibitor. Diabetes. 1997;46:1473-1480.

7. Ishii H, Jirousek MR, Koya D, et al. Amelioration of vascular dysfunctions in diabetic rats by an oral PKC $\beta$ inhibitor. Science. 1996;272:728-731.

8. Danis RP, Bingaman DP, Jirousek M, Yang Y. Inhibition of intraocular neovascularization caused by retinal ischemia in pigs by $\mathrm{PKC}$ $\beta$ inhibition with LY333531. Invest Opbthalmol Vis Sci. 1998; 39(1):171-179.

9. Aiello LP, Clermont A, Arora V, et al. Inhibition of PKC $\beta$ by oral administration of ruboxistaurin is well tolerated and ameliorates diabetes-induced retinal hemodynamic abnormalities in patients. Invest Ophthalmol Vis Sci. 2006;47:86-92.

10. The PKC-DRS2 Study Group. Effect of ruboxistaurin on visual loss in patients with diabetic retinopathy. Opbthalmology. 2006;113: 2221-2230.

11. PKC-DMES Study Group. Effect of ruboxistaurin in patients with diabetic macular edema: thirty-month results of the randomized PKC-DMES clinical trial. Arch Opbthalmol. 2007;125(3):318-324.

12. Gangnon R, Davis MD, Hubbard LD, et al. A severity scale for diabetic macular edema (DME) developed from ETDRS data. Invest Ophthalmol Vis Sci. Published online on June 6, 2008.

13. Early Treatment Diabetic Retinopathy Study Research Group. Photocoagulation for diabetic macular edema. Early Treatment Diabetic Retinopathy Study report number 1. Arch Opbthalmol. 1985; 103:1796-1806.

14. Early Treatment Diabetic Retinopathy Study Research Group. Fundus photographic risk factors for progression of diabetic retinopathy: ETDRS report 12. Opbthalmology. 1991;98:823-833.

15. Barkana Y, Belkin M. Neuroprotection in ophthalmology: a review. Brain Res Bull. 2004;62:447-453.

16. Liu X, Mameza MG, Lee Y, et al. Suppressors of cytokine signaling (SOCS) proteins induce insulin-resistance in the retina and promote survival of retinal cells. Diabetes. 2008;57(6):1651-1658. 